\title{
OSCILLATORY BOTTOM BOUNDARY LAYER UNDER IRREGULAR WAVES
}

\author{
不規則波動下における底面境界屏 \\ Mustafa Ataus SAMAD* and Hitoshi TANAKA** \\ ムスタファアタウス サマド・田中 仁
}

*Member, M. Eng., Research Associate, Dept. of Civil Eng., Tohoku Univ. (Aoba-yama 06, Sendai 980-8579)

${ }^{* *}$ Member, D. Eng., Prof., Dept. of Civil Eng., Tohoku Univ. (Aoba-yama 06, Sendai 980-8579)

Present paper describes characters of bottom boundary layer under irregular waves for both laminar and turbulent flow conditions, assessed through numerical computation. To compute flow phenomena in the boundary layer, firstly, irregular waves have been generated. Then those have been applied at the free surface boundary in Jones and Launder original low Reynolds number $k-\varepsilon$ model. Calculated results indicate that for laminar flow the bottom shear stress is more dominated by high frequency component waves. It has also been observed that high flow inertia close to the bottom produces a double peaked structure in the bottom shear stress in a wave cycle which is unique in irregular waves. The phase difference between bottom shear stress and free stream velocity reduces significantly under turbulent flow condition. A phase difference of about 15 degrees shows a distinct limit between laminar and turbulent regimes. Under turbulent motions, close to the bottom, the turbulent kinetic energy shows a dual dependency on bottom shear stress and on free stream velocity indicating high variability in boundary layer thickness with free stream velocity.

Key Words : Irregular waves, spectrum, bottom boundary layer, turbulence

\section{Introduction}

Bottom boundary layer under waves governs different key parameters that control several physical processes and, as such, it has been the subject of many theoretical and experimental studies. Most of these studies, however, had been restricted mainly to the boundary layers observed with linear harmonic wave motion in the free stream. Among the experimental studies, Jensen ${ }^{1)}$ presented the most recent and detailed investigations, while Sana and Tanaka $^{2)}$ presented a comparative study on the predictive ability of low Reynolds number $k-\varepsilon$ models. A review on the developments in the theories of wave boundary layer can be found in Nielsen". Several researchers had also studied nonlinear cnoidal wave theory, e.g. Nadaoka et al. ${ }^{4)}$, Tanaka et al. ${ }^{5}$, Kawamura et al. ${ }^{6}$, and had contributed towards the understanding of bottom boundary layer characters. Although, some very detailed understanding on near bottom turbulence generation and its dissipation had been achieved through these studies, the results still can not be applied directly to near-shore shallow water regions.

Owing to the randomness of ocean waves, it is of practical importance that the bottom boundary layer character is investigated under irregular waves. However, only few studies on it have been reported in open literature. Madsen et al. ${ }^{7}$ proposed an analytical model to evaluate the spectral wave dissipation based on linearized boundary layer equation, a constant eddyviscosity description and directional wave spectrum. A similar approach was also adopted by Madsen ${ }^{8}$ for spectral wave current combined flow. Myrhaug9) expressed irregular wave 
bottom friction factor considering that the wave motion can be expressed as Gaussian narrowband random process. The underlying assumption that the representative maximum bottom shear stress under irregular wave can be expressed as that of sinusoidal wave, remains the same as that considered by Madsen. All these studies considered representation of integrated irregular wave properties in terms of representative spectral properties and, therefore, did not consider the temporal variation of turbulence structure and bottom shear stress.

Present paper describes the effects of irregular waves on bottom boundary layer under both laminar and turbulent flow conditions analyzed through numerical computation. Here, firstly, the irregular wave time series has been generated from input spectral properties, and then it has been applied to compute flow in the boundary layer through Jones and Launder ${ }^{10}$ original low Reynolds number $k-\varepsilon$ model.

\section{Governing Equations}

\subsection{Irregular Wave}

The spectral density for irregular water surface elevation can be computed using Bretschneider-Mitsuyasu ${ }^{11)}$ spectral density formulation as given by:

$$
\begin{aligned}
S_{\eta}(f)= & 0.257 H_{1 / 3}^{2} T_{1 / 3}\left(T_{1 / 3} f\right)^{-5} \\
& \exp \left[-1.03\left(T_{1 / 3} f\right)^{-4}\right]
\end{aligned}
$$

where, $H_{1 / 3}$ and $T_{1 / 3}$ are significant wave height and period respectively, and $f$ is wave frequency. Applying small amplitude wave theory, following relationship can be obtained for spectral densities of water surface elevation and free stream velocity ${ }^{12)}$ :

$$
\begin{aligned}
S_{U}(f) & =H_{U}{ }^{2}(f) S_{\eta}(f) \\
& =\left(\frac{\omega}{\sinh 2 \pi h / L}\right)^{2} S_{\eta}(f)
\end{aligned}
$$

where, $S_{U}(f)$ and $S_{\eta}(f)$ are spectral densities for velocity and surface elevation respectively, $H_{U}(f)$ is velocity transfer function, $L$ wave length, $h$ water depth, $\omega(=2 \pi f)$ angular frequency and $\mu$ $(=v \rho)$ is dynamic viscosity.

Obtained velocity spectrum has then been used to generate velocity time variation considering that irregular waves can be resolved as a sum of infinite number of wavelets with small amplitudes and random phases ${ }^{13}$. The summation equation stands as:

$$
\begin{gathered}
U(t)=\operatorname{Lim}_{k \rightarrow \infty} \sum_{i=1}^{k} A_{U i} \cos \left(2 \pi f_{i} t+\phi_{i}\right) \\
A_{U i}=2 \sqrt{S_{U}\left(f_{i}\right) \Delta f_{i}}
\end{gathered}
$$

where, $\mathrm{U}(t)$ is instantaneous free stream velocity, $A_{U i}$ velocity amplitudes of component waves, $f_{i}$ component frequencies, $t$ time, $\phi_{i}$ component phases and $\Delta f_{i}$ is frequency increment between successive wave components.

\section{2 k- $\varepsilon$ Model}

Flow in the bottom layer can be computed based on linearized boundary layer equation, given by:

$$
\frac{\partial u}{\partial t}=-\frac{1}{\rho} \frac{\partial p}{\partial x}+\frac{1}{\rho} \frac{\partial \tau}{\partial z}
$$

where, $u$ is velocity in the direction of flow, $p$ pressure, $\tau$ shear stress, $t$ time and $\rho$ is mass density of the fluid. $x$ - and $z$ - are the coordinates along and perpendicular to the direction of flow, respectively. The pressure term is evaluated from the consideration that outside boundary layer the shear stress vanishes and the velocity approaches free stream velocity, $U$. This essentially means that the pressure gradient rcmains constant throughout the boundary layer and can be determined from the free stream velocity variation.

The shear stresses for laminar and turbulent flows are defined based on kinematic viscosity and turbulent eddy viscosity, respectively. These are given by:

$$
\frac{\tau}{\rho}=v \frac{\partial u}{\partial z}
$$

and

$$
\frac{\tau}{\rho}=v \frac{\partial u}{\partial z}-\overline{u^{\prime} v^{\prime}}
$$

where, $v$ is kinematic viscosity and $-\overline{u^{\prime} v^{\prime}}$ is Reynolds stress, expressed as:

$$
-\overline{u^{\prime} v^{\prime}}=v_{t} \frac{\partial u}{\partial z}
$$

where, $v_{t}$ is turbulent viscosity. 
The closure is achieved through expressing the turbulent viscosity in terms of the turbulent kinetic energy, $k$ and its dissipation rate $\varepsilon$, such that,

$$
v_{t}=C_{\mu} f_{\mu} \frac{k^{2}}{\varepsilon}
$$

where $C_{\mu}$ and $f_{\mu}$ are parameters determined from experimental results.

Equations (5) through (9) are solved numerically along with $k$ and $\varepsilon$ transport equations. The later are given as:

$k$-transport equation:

$$
\begin{aligned}
\frac{\partial k}{\partial t}= & \frac{\partial}{\partial z}\left\{\left(v+\frac{v_{t}}{\sigma_{k}}\right) \frac{\partial k}{\partial z}\right\}+v_{t}\left(\frac{\partial u}{\partial z}\right)^{2} \\
& -\varepsilon-D
\end{aligned}
$$

$\varepsilon$-transport equation:

$$
\begin{aligned}
\frac{\partial \varepsilon}{\partial t}= & \frac{\partial}{\partial z}\left\{\left(v+\frac{v_{t}}{\sigma_{\varepsilon}}\right) \frac{\partial \varepsilon}{\partial z}\right\}+C_{1} f_{1} v_{t} \frac{\varepsilon}{k}\left(\frac{\partial u}{\partial z}\right)^{2} \\
& -C_{2} f_{2} \frac{\varepsilon^{2}}{k}+E
\end{aligned}
$$

where, $\sigma_{k} D, \sigma_{v} C_{l}, f_{1}, C_{2}, f_{2}$ and $E$ are model parameters.

In the present computation Jones and Launder original $k-\varepsilon$ model has been used. The selection of this particular model has been based on the testing of low Reynolds number $k$ $\varepsilon$ models by Sana and Tanaka ${ }^{2}$. For numerical computation the governing equations have been made non-dimensional and a Crank-Nicolson type implicit finite difference scheme has been applied. In the non-dimensional form of the governing equations, along with free stream velocity variation only the Reynolds number $\left(R e_{1 / 3}\right)$ and the Strouhal $(S)$ number are required to compute flows in laminar, turbulent or in transitional regimes. The representative Reynolds number is introduced later in section 2.3 and the Strouhal number is defined as:

$$
S=\frac{U_{1 / 3}}{\omega_{1 / 3} z_{h}}
$$

where, $U_{1 / 3}$ is significant free stream velocity, $\omega_{1 / 3}$ significant angular frequency and $z_{h}$ is an arbitrary normalizing depth. Further details of the modeling system can be found in Tanaka and Sana ${ }^{14)}$.
For laminar motion it is also possible to compute spectral density function for bottom shear stress by applying small amplitude wave theory ${ }^{15}$. The bottom shear stress spectral density and transfer function are given by:

$$
\begin{aligned}
S_{\tau}(f) & =H_{\tau}{ }^{2}(f) S_{\eta}(f) \\
& =(\sqrt{2} \beta \mu)^{2} H_{U}{ }^{2}(f) S_{\eta}(f)
\end{aligned}
$$

and

$$
\begin{gathered}
H_{\tau}(f)=\sqrt{2} \beta \mu \frac{\omega}{\sinh k h} \\
\text { with } \beta=\sqrt{\frac{\omega}{2 v}}
\end{gathered}
$$

where, $S_{\tau}(f)$ and $H_{\tau}(f)$ are bottom shear stress spectral density and transfer function, respectively. From $k-\varepsilon$ model result the transfer function can be computed as, $H_{\tau}^{2}(f)=S_{\tau}(f) / S_{\eta}(f)$ and $S_{\eta}(f)$ can be evaluated using Eq.(2).

\subsection{Representative Reynolds number}

The wave Reynolds number for sinusoidal waves $(R e)$ as given by Jonsson ${ }^{16)}$ is:

$$
\operatorname{Re}=\frac{U_{0} a_{m}}{v}=\frac{U_{0}^{2}}{v \omega}
$$

where $U_{o}$ is free stream velocity amplitude and $a_{m}$ is maximum bottom orbital displacement.

The irregular wave Reynolds number has been defined in terms of significant wave properties and analogous to $R e$, given by:

$$
\operatorname{Re}_{1 / 3}=\frac{U_{1 / 3}{ }^{2}}{v \omega_{1 / 3}}
$$

with

$$
\omega_{1 / 3}=\frac{2 \pi}{T_{1 / 3}}
$$

where $T_{1 / 3}$ is significant wave period.

\section{Results and Discussions}

Test runs have been carried out to cover both laminar and turbulent flow regimes. Spectral properties from laminar computation results have been compared with analytical solutions to validate model performance. Then the simulation has been extended to turbulent computation.

The input wave parameters specified for laminar computation have been the significant 
wave height, $H_{1 / 3}=50 \mathrm{~cm}$ and significant wave period, $T_{1 / 3}=10 \mathrm{sec}$ with a water depth $(h)$ of $10 \mathrm{~m}$. An arbitrary normalizing depth value of $z_{h}=100 \mathrm{~cm}$ has also been used to amplify the results within the boundary layer. In order to facilitate comparison between laminar and turbulent flow properties, generated laminar velocity time series has been multiplied to produce flow in the turbulent region. This has resulted in a significant wave height of, $H_{1 / 3}=500 \mathrm{~cm}$. The corresponding Reynolds numbers $\left(R e_{1 / 3}\right)$ in laminar and turbulent regions are $7.28 \times 10^{4}$ and $7.28 \times 10^{6}$ respectively.

\subsection{Irregular Wave Simulation}

Simulation of irregular wave has been performed to generate sufficiently long time series to use in the $k-\varepsilon$ model. Number of waves thus generated were over 175 to facilitate computation of spectral properties. A total of 250 component waves have been considered for which randomly generated frequencies have been used ${ }^{13)}$.

The accuracy of generated velocity data has been checked against the input spectrum. Figure 1 shows the comparison of input (Eq.(2)) and generated velocity spectra. It can be seen from the figure that the generated velocity spectra is in very good agreement with the input spectra. The wave energy is spreaded in the frequency range of $0.05 \mathrm{~Hz}$ to $0.40 \mathrm{~Hz}$ or equivalently within the period of $2.5 \mathrm{sec}$ to $20.0 \mathrm{sec}$.

\subsection{Laminar boundary layer properties}

$k$ - $\varepsilon$ model result for laminar computation has

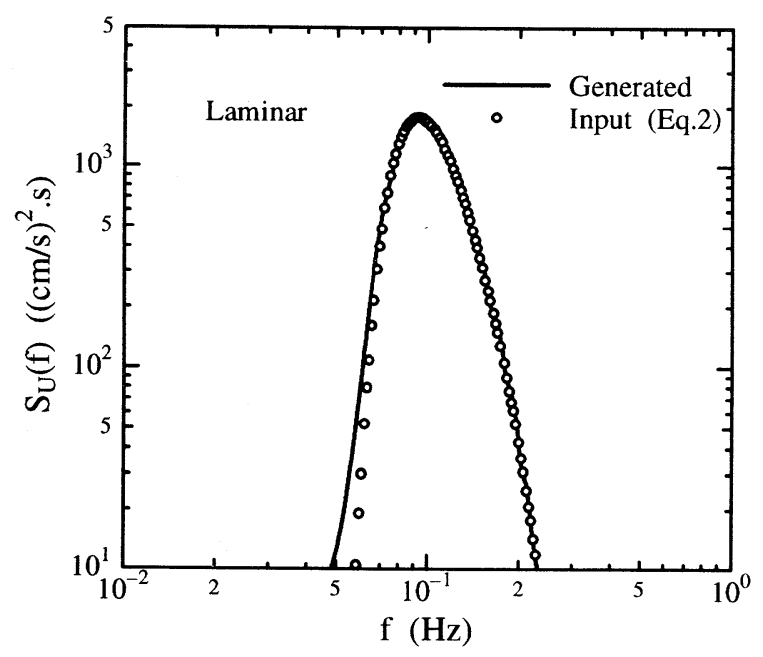

Fig.1: Input and generated wave velocity spectral density. first been compared with the spectral properties obtained analytically. Figures 2 and 3 show the comparison of spectral density and transfer function of bottom shear stress from simulated results with those from analytical solutions (Eqs.(13) and (14)). Both the figures show reasonably good agreement with computed results, however, the simulated transfer function is slightly under estimated. Although bottom shear stress spectral density shows similar trend as that observed in velocity spectrum, in Fig. 3 it can be seen that the time variation of bottom shear stress is more dominated by high frequency component waves. As such the peak of the transfer function appears at a higher frequency $(f=0.12 \mathrm{~Hz})$ than that corresponding to significant wave period $(f=0.1 \mathrm{~Hz})$.

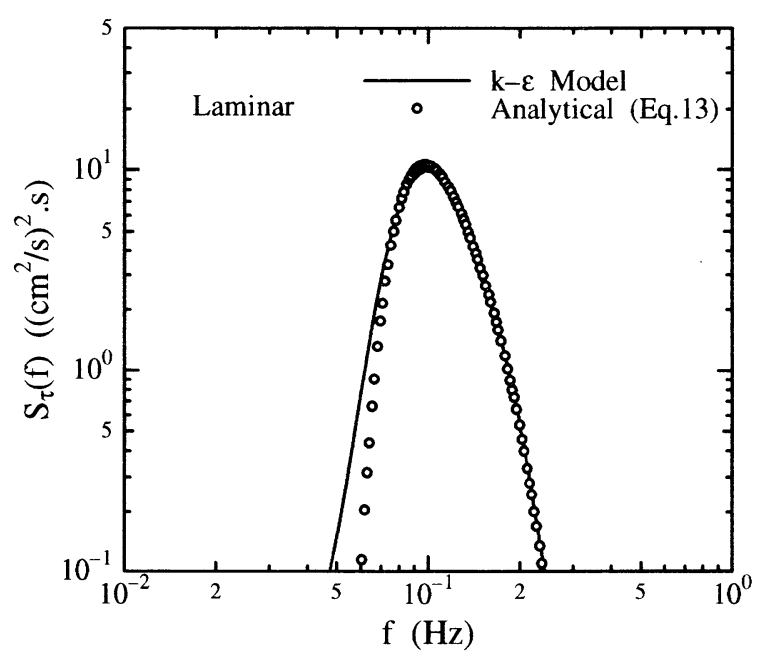

Fig.2: Bottom shear stress spectra from generated data and $k$-E model result.

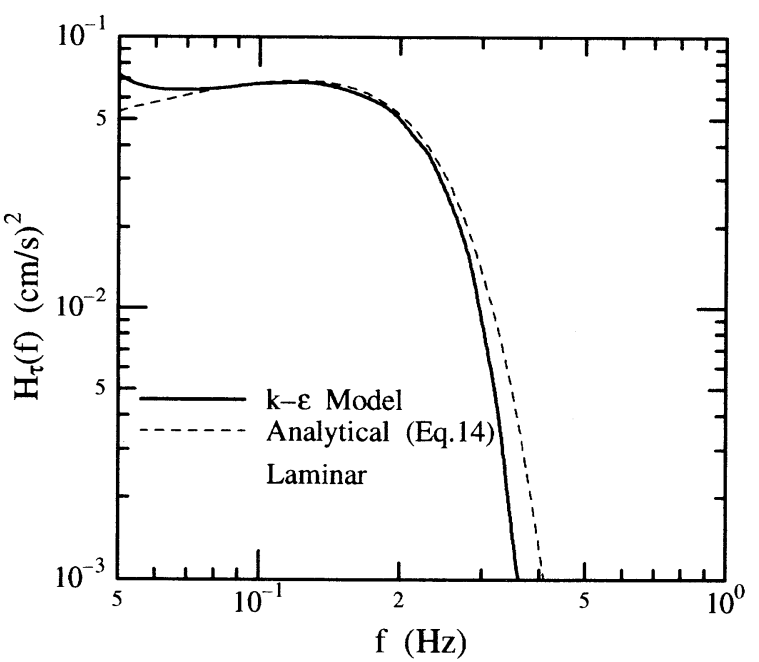

Fig.3: Comparison of bottom shear stress transfer functions. 
Computed time variation of bottom shear stress and corresponding free stream velocity variations are shown in Fig.4. Generally the domination of high frequency wave components can easily be visualized comparing bottom shear stress peaks with that of the velocity. A few interesting variations in the bottom shear stress can be observed at locations marked with an arrow in the figure. The bottom shear stress is dependent on flow acceleration and inertia. This contributes to occasional appearances of a second peaks in a wave cycle as can be seen in wave W3. At smaller velocity magnitudes it cycles $\mathrm{W} 1$ or $\mathrm{W} 4$ the shear stress shows a further loop indicating a reversal in bottom shear stress although the free stream velocity remains unidirectional. Wave W3 shows a 'heart' like shape representing a double peaked structure in bottom shear stress as discussed before.

Vertical velocity distribution at selected phases are shown in Fig.7. It is observed that at similar free stream velocity magnitudes the vertical velocity distribution is much different in two opposite phases. It is clearly seen between phases $\mathrm{A}$ and $\mathrm{E}$ or between $\mathrm{B}$ and $\mathrm{F}$. Although

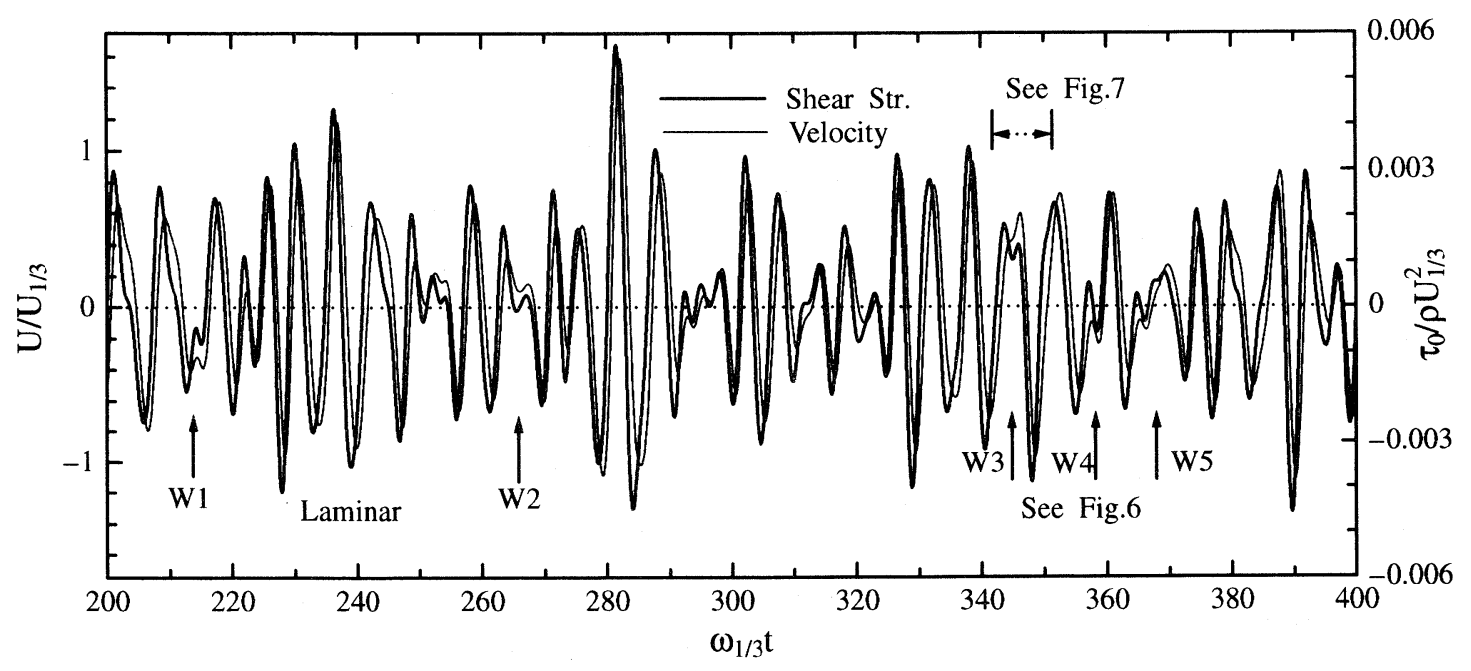

Fig.4:Simulated velocity and bottom shear stress for laminar flow.

even causes reversal in bottom shear stress where no such change can be seen in free stream velocity (wave W4 and W5).

Similar variation in bottom shear stress has been observed under cnoidal waves as reported by Tanaka et al. ${ }^{5)}$ and is shown in Fig.5. It can be seen that due to high flow deceleration and inertia, a trough peak appears in the bottom shear stress at $t / T=0.070$ while no such peak can be seen in the free stream velocity.

Figure 6 further illustrates this complicated behavior of bottom shear stress for individual waves marked in Fig.4 along with that for corresponding sinusoidal wave. Here the bottom shear stress is correlated with free stream velocity variation. Such a correlation of bottom shear stress for sinusoidal wave follows an elliptic path as can be seen in the figure. However, for irregular wave when these quantities are correlated in an individual wave cycle, the behavior is much different. For wave the free stream velocity magnitude is different between $C$ and $G$, the difference in velocity overshooting can easily be visualized.

\subsection{Turbulent behavior in the boundary layer} Generation of turbulent velocity has been

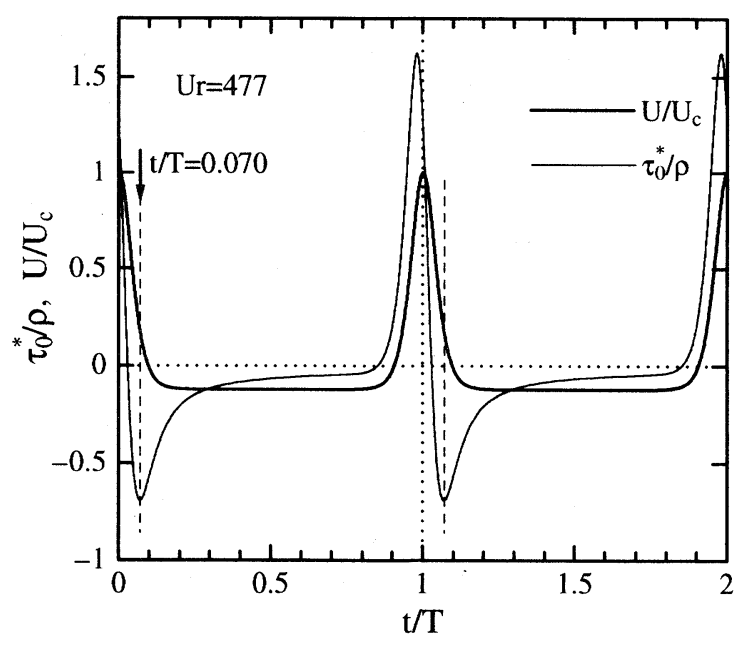

Fig.5: Bottom shear stress variation with free stream velocity under cnoidal wave. 


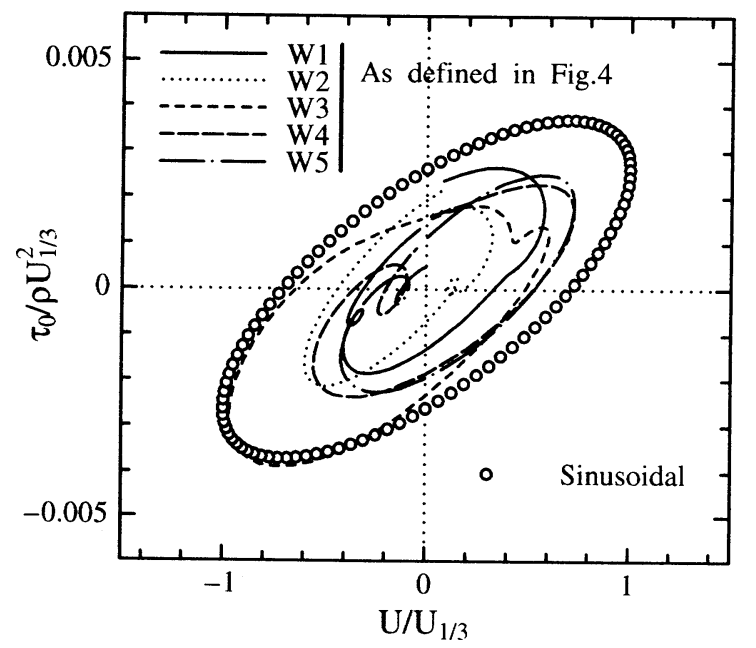

Fig.6: Bottom shear stress and free stream velocity correlation under laminar flow.

achieved by multiplying the generated laminar velocity time series. This has been done mainly to facilitate comparison between laminar and turbulent flow characteristics.

Time variation of free stream velocity and corresponding bottom shear stress are presented in Fig.8 along with the variation in turbulent kinetic energy, $k$. Here the bottom shear stress shows a more regular variation unlike as observed for laminar flow. The near bottom turbulence dissipation significantly reduced the flow inertial effects and, therefore, no reversal in bottom shear stress can be observed. The phase difference is also much reduced making the

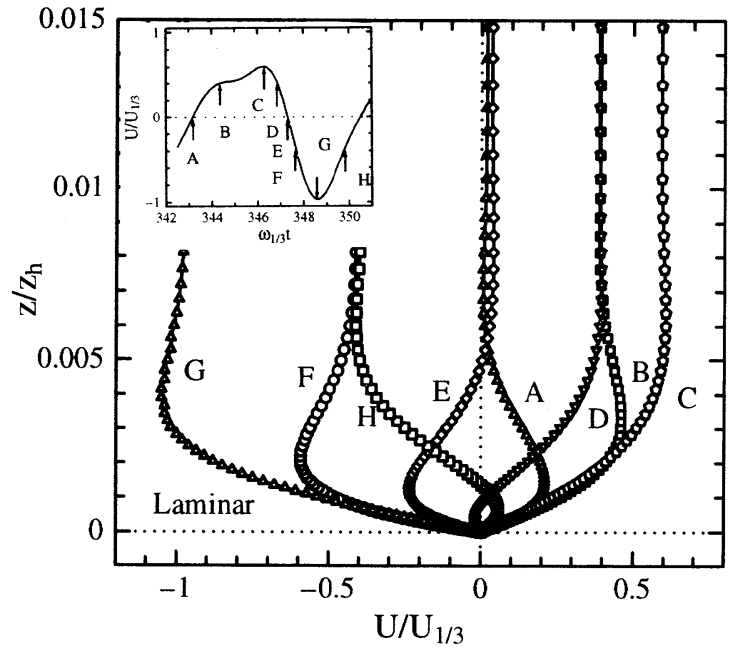

Fig.7: Vertical velocity distribution: Laminar flow.

shear stress almost simultaneous with free stream velocity.

Figure 9 shows the correlation of bottom shear stress with free stream velocity for individual waves as indicated in Fig.8. The bottom shear stress shows a rapid variation with free stream velocity similar to that from a power function. Due to the decrease in phase difference it also has a much narrow band extent.

The vertical velocity distribution is presented in Fig.10. Turbulence mixing causes higher velocities close to the wall, and subsequent reduction in flow inertia significantly

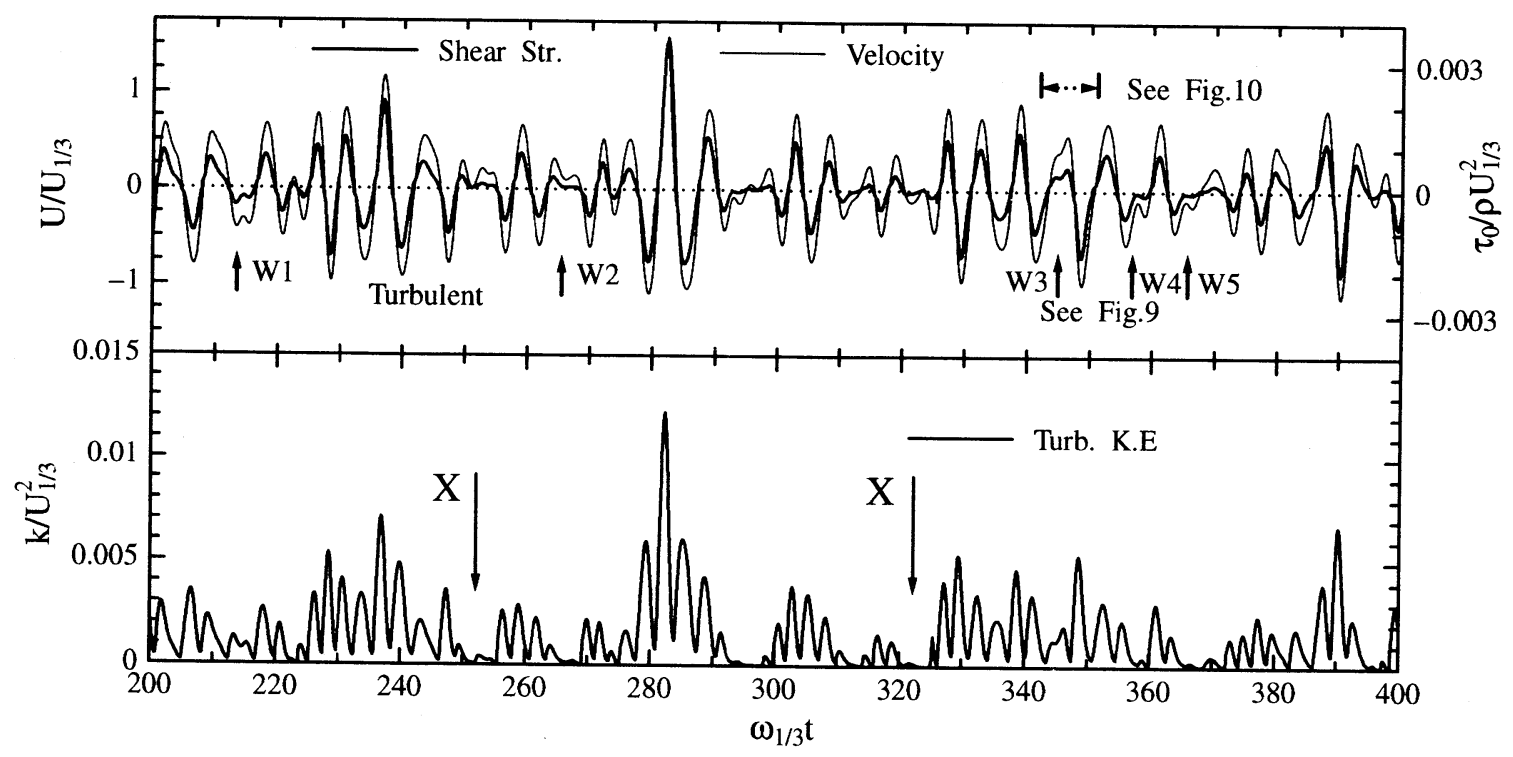

Fig. 8: $\quad$ Simulated turbulent velocity and bottom shear stress. 
reduces velocity overshooting as can be seen in the figure. However, the effect of flow inertia can still be observed between phases B, F and between $\mathrm{D}, \mathrm{H}$ having similar free stream velocity magnitudes.

Figure 8 also shows the time variation of turbulent kinetic energy at Stokes layer thickness from the bottom. Stokes layer thickness $\left(\delta_{l}\right)$ for irregular wave has been defined as:

$$
\delta_{l}=\sqrt{\frac{2 v}{\omega_{1 / 3}}}
$$

In Fig.8, bursts in turbulent kinetic energy can be seen corresponding to peaks or troughs in velocity or bottom shear stress. At higher velocity magnitudes it is observed to be in phase

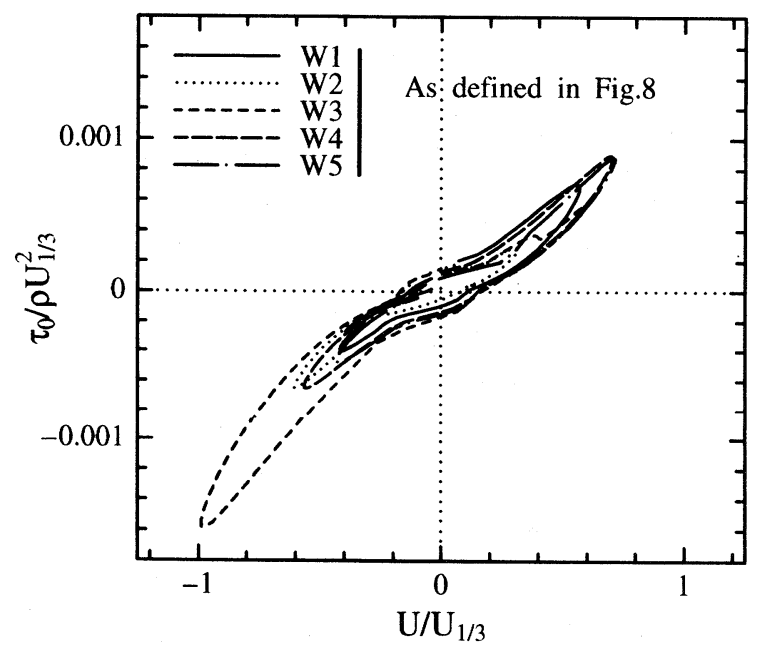

Fig.9: Bottom shear stress and free stream velocity correlation under turbulent flow.

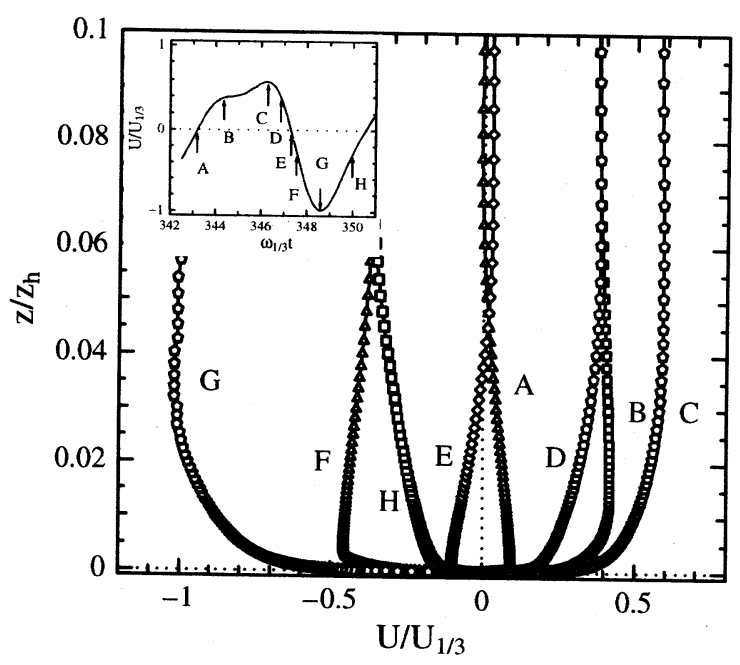

Fig.10: Vertical velocity distribution: turbulent flow. with free stream velocity variation. But when the bottom shear stress is much smaller for a prolonged period (as indicated at locations marked with an X), the turbulent kinetic energy closely corresponds to bottom shear stress. Also it results in a very small turbulent kinetic energy value like in the case of laminar flow. When the turbulent kinetic energy is compared with free stream velocity variation and with bottom shear stress, as shown in Fig.11 and Fig.12 respectively, the phenomena of dual dependence can be clearly observed. It can be seen that the turbulent kinetic energy is closely correlated with velocity at higher values of $k$ (Fig.11) and it shows excellent correlation with bottom shear stress at smaller values (Fig.12). The reason could be traced to the thickness of bottom boundary layer, which changes continuously

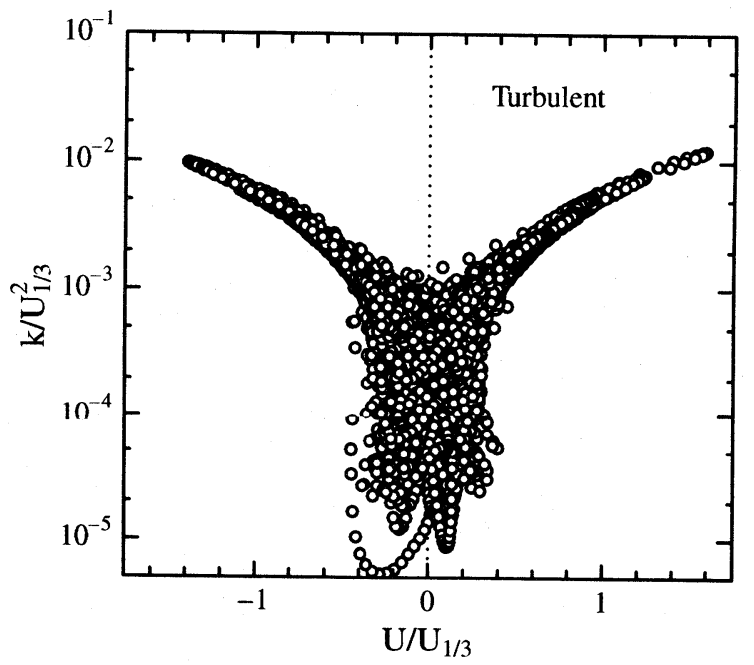

Fig.11: Variation of turbulent kinetic energy with free stream velocity.

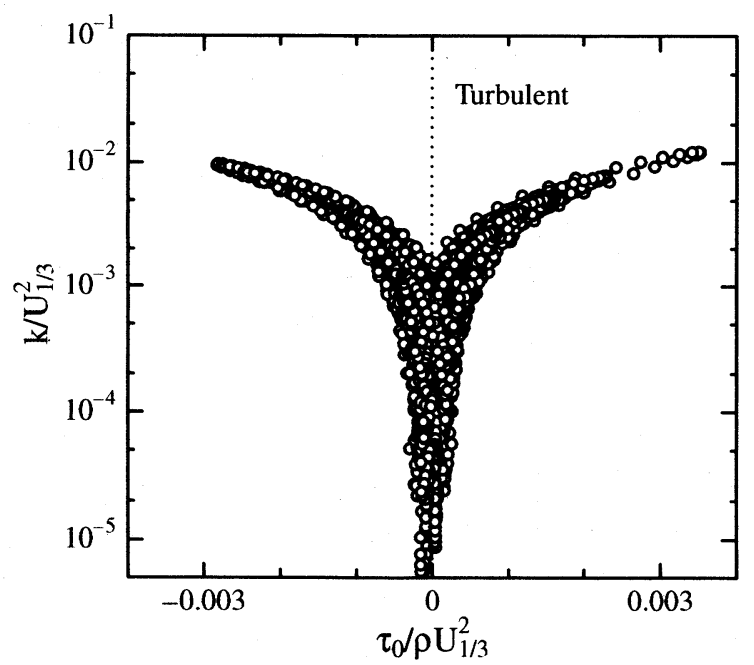

Fig.12: Variation of turbulent kinetic energy with bottom shear stress. 


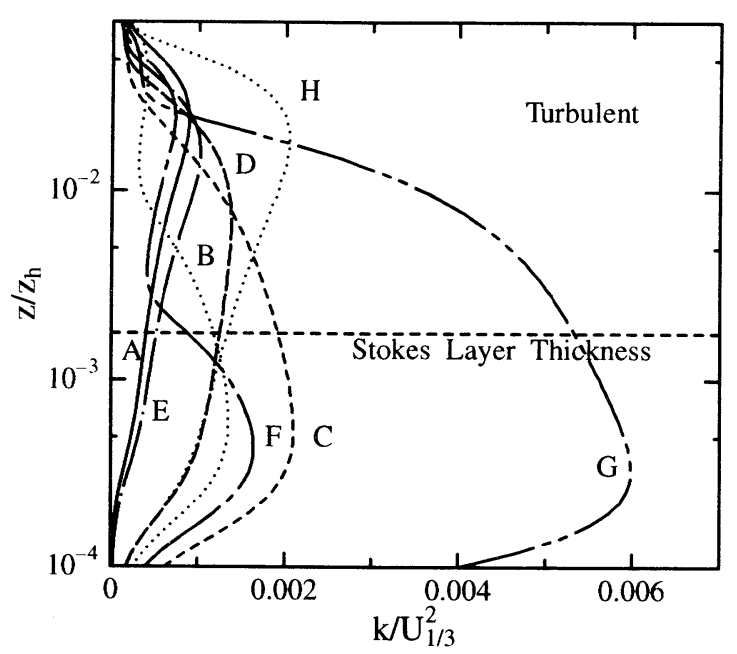

Fig.13: Vertical profiles of turbulent kinetic energy at selected phases.

under the variation in free stream velocity. When the Stokes layer thickness is located outside boundary layer turbulent energy is closely related to free stream velocity. On the other hand, kinetic energy values within boundary layer thickness are more dependent on the bottom shear stress.

Figure 13 shows the vertical profiles of turbulent kinetic energy at selected phases. High turbulent kinetic energy values can be observed corresponding to high free stream velocity magnitudes. During deceleration generally higher turbulence can be seen outside the boundary layer thickness.

It can be observed in Figs. 4 and 8 that, the change in phase angle between velocity and shear stress is continuous and dependent on velocity gradient. The phase difference at the crest of individual waves for both laminar and turbulent regimes is shown in Fig.14. Here the phase difference $(\theta)$ is presented against the nondimensional crest velocities $\left(U_{c} / U_{1 / 3}\right)$. As mentioned before, in the turbulent regime turbulent dissipation reduces the phase difference significantly. It can be seen in the figure that a phase difference of about $15^{\circ}$ constitutes the limit between the laminar and the turbulent flow regimes. The average values of phase difference in laminar and turbulent regimes are about $35^{\circ}$ and $8^{\circ}$ respectively. For laminar flow it is considerably smaller than that of corresponding sinusoidal wave.

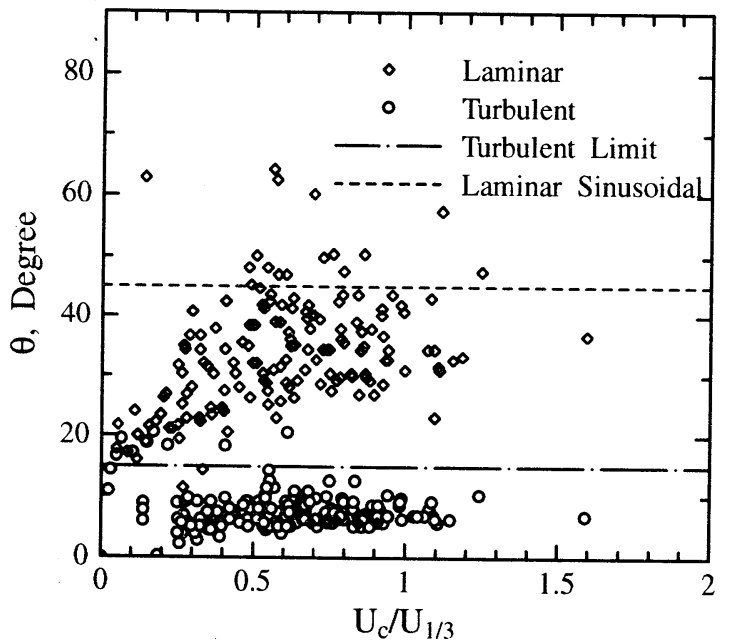

Fig.14: Phase differences for laminar and turbulent flows.

\section{Conclusions}

The oscillatory boundary layer behavior under irregular wave motion has been investigated for both laminar and turbulent flow regimes through numerical computation. The irregular wave velocity time series has first been generated and later specified at the free stream boundary for flow computation in the boundary layer. The computation has been performed through Jones and Launder original low Reynolds number $k-\varepsilon$ model.

The generation of irregular wave velocity shows a satisfactory result when compared with input spectral properties. Reasonably good agreement has also been obtained for spectral properties of $k$ - $\varepsilon$ model results when compared with those from analytical solutions for laminar flows.

Under laminar motion, it has been observed from model result that the bottom shear stress is dominated more by high frequency component waves. In physical terms it might be explained as the influence of flow inertia close to the bottom. This also causes a double peaked structure and reversal in bottom shear stress, whereas no such phenomena can be seen in free stream velocity.

Turbulent mixing causes significant reduction in velocity overshooting close to the bottom and reduces the phase difference 
between bottom shear stress and free stream velocity.

Thickness of bottom boundary layer varies considerably with the randomness in free stream velocity variation. While the turbulent kinetic energy is strongly related to bottom shear stress inside the boundary layer, it is more dependent on the free stream velocity outside.

Phase difference at the peaks of individual waves shows a distinct limit for turbulent flow at about 15 degrees. The average difference for laminar flow is considerably smaller than that from sinusoidal waves.

\section{Acknowledgment}

A part of the study was financially supported by Grant-in-Aid of the Ministry of Education, Science and Culture, Japan, to whom the authors would like to express their sincere appreciation.

\section{REFERENCES}

1) Jcnsen, B.L.: Experimental investigation of turbulent oscillatory boundary layers, Inst. Hydrodyn. Hydraul. Eng., Technical Univ. of Denmark, Lyngby, Series Paper 45, pp.157, 1989.

2) Sana, A. and Tanaka, H.: The testing of low Reynolds number $k$ - $\varepsilon$ models by DNS data for an oscillatory boundary layer, Flow Modeling and Turbulence Measurement VI, pp.363-37.0, 1996.

3) Nielsen, P.: Coastal bottom boundary layers and sediment transport, World Scientific Publishing Co., Singapore, 1992.

4) Nadaoka, K., Yagi, H., Nihei, Y. and Nomoto, K.: Characteristics of turbulent structure in asymmetric oscillatory flow, Proc. Coastal Eng. JSCE, Vol.41, pp.141145, 1994. (in Japanese)

5) Tanaka, H., Sumer, B.M. and Lodahl, C.: Theoretical and experimental investigaiton of laminar boundary layers under cnoidal wave motion, Coastal Eng., 1998. (in press)

6) Kawamura, I., Tanaka, H., Yamaji, H. and Sana, A.: Transition to turbulence in an asymmetric oscillatory boundary layer, Proc. Coastal Eng., JSCE, Vol.44, pp.136-140, 1997. (in Japanese)

7) Madsen, O.S., Poon, Y.K. and Graber, H.C.: Spectral wave attenuation by bottom friction: Theory, Proc. 32nd Int. Conf. Coastal Eng., pp.492-504, 1988.

8) Madsen, O.S.: Spectral wave-current bottom boundary layer flows, Proc. 38th Int. Conf. Coastal Eng., pp.384-398, 1994.

9) Myrhaug, D.: Bottom friction beneath random waves, Coastal Eng. Vol.24, pp.259273. 1995.

10) Jones, W.P. and Launder, B.E.,: The prediction of laminarization with a two equation model of turbulence, Int. J. Heat and Mass Transfer, Vol.15, pp.301-314, 1972.

11) Mitsuyasu, H.,: On the growth of spectrum of wind-generated waves, Proc. 17th Jap. Conf. Coast. Engg., pp.1-7, 1970. (in Japanese)

12) Horikawa, K.: Nearshore dynamics and coastal processes: theory, measurements and predictive models, Univ. of Tokyo Press, 1988.

13) Goda, Y.,: Numerical experiments on wave statistics with spectral simulation, Rep. of Port \& Hour. Research Inst., Vol.9, No.3, pp.3-57. 1970.

14) Tanaka, H. and Sana, A.,: Numerical study on transition to turbulence in a wave boundary layer, Sed. Transport Mechanism in Coast. Env. and Rivers, pp.14-25, 1994.

15) Mitsunobu, N. and Sato, S.: Representative velocity and shear stress under irregular waves, Proc. 44th Annual Meeting, JSCE, Vol.2, pp.770-771, 1989. (in Japanese)

16) Jonsson, I.G.: Wave boundary layers and wave friction factors. Proc. 10th Int. Conf. Coastal Eng., pp.127-148, 1966.

(Received April 24, 1998) 